\title{
Design \& Development of Water Management System
}

\author{
N Ramarao \\ Asst Professor, \\ Department of Electrical \& Electronics Engineering \\ BMS Institute of Technology \& Management \\ Bangalore, Karnataka,India \\ Gokul Manikanta T \\ UG Student, \\ Department of Electrical and Electronics Engineering \\ BMS Institute of Technology \& Management \\ Bangalor, Karnataka, India
}

\author{
Girija S \\ UG Student, \\ Department of Electrical and Electronics Engineering \\ BMS Institute of Technology \& Management \\ Bangalore, Karnataka, India \\ Shiva Kumar K R \\ UG Student, \\ Department of Electrical and Electronics Engineering \\ BMS Institute of Technology \& Management \\ Bangalore, Karnataka, India
}

\author{
Vinod P \\ UG Student, \\ Department of Electrical and Electronics Engineering \\ BMS Institute of Technology \& Management \\ Bangalore, Karnataka, India
}

\begin{abstract}
Undoubtedly, water is one of the most important resources on entire globe. No one including human beings, animals, plants and insects can live without water. Water is a scarce resource and it may deplete over coming years due to overuse. The bad quality, overflowing from tanks, leakage in pipes and inefficient usage of water are the main cause which leads to the wastage of water. So it is necessary to have control on water wastage and usage as well by introducing or building a system which will overcome the water wastage related issue using Internet of Things(IOT).
\end{abstract}

Keywords:- Sensors, cloud storage, real time monitoring, microcontroller, Internet of Things (IOT).

\section{INTRODUCTION}

Recent development of Infrastructure, increase in population, leakage in pipes, uncontrolled usage and wastage of water, pollution, etc leading to scarcity of water is faced by human beings. So it is necessary to find the alternative system which can help to reduce the water wastage issues for which Internet of Things (IOT) is the solution which helps in building an automated system for real time water monitoring. Embedding different sensors with a micro controller we can create such a system using the cloud services for data storage.

As there is an uneven distribution of water across the city an automated system must be developed so that the water is distributed equally with equal pressure to the residents in the city. For checking the water quality the parameters like $\mathrm{Ph}$, turbidity, temperature, TDS, etc. must be calculated. Level sensors are used to check the water level in tanks, pressure, flow sensors can be used to detect the leakage in pipes. The design of a water level sensor device is able to detect and control the level of water in a certain water tank. The system firstly senses the amount of water available in the tank by the level detector part and then adjusts the state of the water pump in accordance to the water level information.

There has been wastage of water daily through the pipeline leakages due to it full water were never arrived to the taps. The existing system are still lacking abilities to detect accurate estimation of water leakage in water tanks. The aims are to perform a small-scale study of existing pipeline leakage detection system, to develop a real-time prototype pipeline leakage alert system and to validate the proposed prototype through experimentation. Mobile phone can be configured as the alerts transmitter of the system to the user in case of water leaks.

\section{LITERATURE REVIEW}

\section{A. Water Leakage System Using IOT [1]}

In this paper, the flow of water through the domestic pipeline can be monitored, forecasted and visualize from anywhere in the world using internet through computer or smartphone. The collected data can be analyzed for making predictions to the users and also for demand management, asset management and leakage management. With the water as flowing liquid the system was tested successfully. The work can be extended to forecast data for larger communities with customer satisfaction involving low cost and better performance of the overall system.

According to the author of the paper, the proposed model to forecast and monitor the consumption of water basically consisted of flow meter, micro controller and cloud infrastructure. Hall effect based flow meter was used to measure the flow rate of the water and Arduinouno and Raspberry Pi acted as microcontroller based devices. The flow meter measured the flow rate of the water and generated a pulse signal accordingly. The flow meter was 
wired with arduino so as to sense the pulses from flow meter. The raspberry $\mathrm{Pi}$ which is a microcomputer receives the data from arduino microcontroller which is connected to flow meter and was programmed to read the arduino signal, process the data and store in raw data files. The raspberry $\mathrm{Pi}$ was programmed such that it processed the raw data and uploaded them into the web server. In order to process the request from large number of customers cloud interfacing was initialized. The end users via web interface were able to visualize the data. The data from the database would then be utilized by data prediction algorithm for making predictions as per the users. The request for the prediction comes from the users via the web interface.

So this paper provided us the result showing that the alert message would be sent after being recognized at certain level and then the user would acknowledge the message and act with actions. An experiment testing was conducted to see the results of how geophone sensor worked which was set up at the edge point of pipe which was connected to the circuit.

\section{B. Smart Water Conservation and Management system using IOT.[2]}

This paper has given a brief discussion about smart water supply across a particular area by harnessing the technological usage of IOT concept, simultaneously improving the water quality of the drinking water. This idea could be implemented by various government across the world to save water bodies from drying up caused by excess water usage. This system could also be implemented to reuse the water and also prevent water wastage. Purification and conductivity test of water also played a huge role in the welfare of public which could reduce the overall disease caused due to deficiency, thus creating a healthier society of people. This system was also further enhanced by including modules which improved the given situation further in order to prevent the excess usage of water and saving underground water. Water conductivity sensors were used in water-quality applications to measure how well a solution conducts an electrical current. This type of measurement assesses the concentration of ions in the solution. The more ions that are in the solution, the higher the conductivity, hence higher the impurities. The author also used a mineral cartridge to replenish the mineral content of the purified water from the substation. But the mineral cartridge expires due to the usage of minerals present in it. They took a survey based upon the mineral content intensity present in the tank of water and observed a constant decrease in mineral content over time and the mineral content depleted to zero after 4 months of time. Hence an intimation was sent to the user interface which warned the user to replace the mineral cartridge.

Mineral cartridges are used to control the mineral content of the purified water in substation. After the purification process, the water mineral content is reduced. This water is good enough for both commercial and industrial purposes but not apt for drinking and cooking purposes. Hence mineral cartridges are used at house level to replenish the mineral contents lost during purification processes. As said before, the use of mineralized water is way too low when compared to commercial purpose. Thus the installation of the mineral cartridge is based on specific needs and requirements. Thus by using model designed as per the author we can monitor the quantity and quality of the water as well as improve it by various controlled purification systems such as UV and RO processes and mineral replenishment.

\section{An Internet of Things Based Model For Smart Water Distribution with Quality Monitoring [3]}

Different sensors such as $\mathrm{pH}$ sensor, conductivity Sensor, temperature sensor are used for monitoring the water quality in this survey. The values from the sensor are uploaded to cloud by the raspberry PI controller through Internet channel at random interval. The reason for choosing the randomness is to avoid all the devices firing the request at the same time. These values can be monitored location wise in real time. If the supplied water is fresh and passed all the tests, even though water reaching to end points is contaminated then we can detect the source of problem e.g. if the supplied water is fresh but water reaching to end point is contaminated then we can find till which region or end point the water reaching is fresh and not contaminated, its means till that point there is no problem, the problem is thereafter.

Using this technique, we can monitor each end point for adequate water supply and immediately a necessary step can be taken for any type of problem. By this technique, we have control over each end point rather than having water valve controls society or area wise in the traditional system. The advantage of having control over each end point is that if any end point doesn't receive the water with adequate pressure, we can supply the water to that end point at a different time by turning off all other control valves within that area or society. Another advantage of this System is that we can turn off control valve when the predefined liters of water is supplied to the specific end point. In this paper, the model of smart water distribution with water quality monitoring is presented. The proposed system is created with the use of different sensors, Raspberry Pi as controller and Cloud for storing the data from Raspberry Pi and sending the command to raspberry PI for measuring water quality and controlling water distribution. The generated data can be viewed using web interface all over the world. The advantage of the system is to provide the adequate water supply with pressure and good quality water to each house, industry, and others. The proposed model can be implemented as a part of the smart city.

In future, proposed system can be made fully autonomous by embedding the artificial intelligence with some predefined set of rules and standard. With the use of Artificial intelligence, smart water distribution can be carried out automatically without human intervention. If any problem is detected in water quality, the system will automatically send a notification to remotely handled devices to carry out necessary steps by the authorized person or dedicated authorities. As per predefined interval, the proposed model generates data and sends it to cloud. Hence, proposed model generates a huge amount of data in the cloud. Therefore, some big data processing framework such as Hadoop framework will be required for analysis of 
generated data, for obtaining necessary information and generating the set of rules for training AI.

\section{Analysis, Design and Development of an IOT Based Water Management System for Residence [4]}

This paper describes a water management system design and construction details. Tank 1 from the reserve tank

automatically turns the pump motor on / off. Tank 1 has two solenoid valves which automatically flows water in two ways. Go to one water filter tank and go to filter water and another tank2. Tank2 has a heater that on by pressing mobile apps button when it needs and turned off automatically, the temperature reaches 35 degrees Celsius and is displayed on the LCD display. It always gives normal water without pressing the mobile app on button. Using Arduino successfully using $\mathrm{C} / \mathrm{C}++$ language, the program automatically allows the water level to be automatically turned on / off by sensing, stopping temperature, WiFi connected with mobile applications. As a conclusion, the system designed in this work is well performed.

There are some limitations of this system that is

(a) There are no features for water quality measurement,

(b) Water cooling properties are absent in this project.

In future, simulating this system there are several improvements can be made in order to upgrade the features such as-

(a) Temperature increment/decrement features can be added,

(b) $\mathrm{pH}$ meter can be used to determine the water quality.

The water level sensor in the water tank1 is submerged; it understands the high and low level of water and sends the data signal which connects the Arduino digital pin 2, 3, 4, 5. Then the water pump switch on the reserve tank based on the sensing of the water level reading which switching the relay module for the on / off switch and then switches on the relay module swing valve for water flowing on / off. Water pump connect to the ardino digital pin 12. Wifi Module's pin Arduino connectsthe digital pins 0, 1 for wireless communications. It connects the mobile app. pressing the button on the mobile app sent for the water heater which connects to the arduino digital pin 13 and the system instructions

were sent. An IC LM 35 is used for the temperature sensor project, which takes temperature from the current water temperature, which interacts on the Adobe board's analog pin $\mathrm{A} 0$, it creates an $\mathrm{ADC}$ that converts the reading to display in LCD which is connected to the pin with Arduino analog pin A4, A5 and then the relay module connected heat switching to 35 degrees Celsius. Open/Close the solenoid valve connect digital pin 11 which automatically in the flowing water. It always gives normal water without pressing the button on mobile applications. Water filter from the water tank 1

and flow of the water is supplied depend on use of water.

Using the workflow author has done this project very smoothly because it is a serial process. For solving any problem or for any system development, the whole work should be handled in that segment so that accuracy can be provided. So, we have followed the workflow to increase accuracy. Due to the six-stage features and feedback scope system, this system used workflow. This workflow feature can return it to the previous step. We can return and correct the system at any time according to the requirement. In this workflow, before completing the next stage, each stage must be completed completely. Such a workflow is basically short and no uncertain requirements for the project. At each stage of determining whether the project is going on in the right direction and whether to continue or cancel the stage.

\section{E. An IOT Based Efficient Water Management System} [5]

In this work, an IoT based efficient water management system, measures the water levels and water quality in storage tanks. It also manages water dispensation and report generation through an user interface. The system also allows the water source at a particular location to be managed and monitored remotely avoiding water wastage and in turn, the water crisis. The whole system is tried out in a closed setup at present. The system will be implemented on a real scenario to test its whole effectiveness for the smart city setup. Internet of Things (IoT) is a happening technology which connects all objects in world to internet so that they can sense, communicate and share information interconnected over public or private network . This work aims to use IoT to develop an efficient water management system. The proposed system attempts to manage all the storage tanks and provide real time monitoring of water levels and water quality through a web application. It also integrates an alert system along with water distribution. It has sensors to sense the data, microcontroller to process the sensed data and some platform tools to store and display them. This paper describes the prototype system design, implementation and description of the tools and technologies of the IoT based efficient water management system. The next section gives a review of the existing literature.

The architecture of the proposed system consists of four layers. They are,

Physical layer: It consists of sensors to measure the water levels and water quality. At this layer, data is collected from sensors and sent to the control unit.

Network layer: It gets the sensor information and processes it using Raspberry Pi.

Administration layer: Data processed by the microcontroller is published to the cloud for storage and analysis.

Application layer: It is the client application that integrates with the cloud and helps to visually manage the system through an user interface.

The sensors are placed in all storage tanks of the city. The ultrasonic sensor measures the water levels whereas the $\mathrm{PH}$ sensor, turbidity sensor and conductivity sensor measure the water quality. Once the data is sensed by the sensors, it is processed and sent to the cloud. Carriots, an IoT platform streams the data from the control unit in JavaScript Object Notation (JSON) format which stores and analyses the data stream .It also allows to perform events, triggers, alarms and so on, as a response to the received data stream. All the water management activities are done through

the client web application with the help of Freeboard. It is a dashboard that acts as a data visualization tool. It receives the data stream from Carriots in JSON format by the data 
source configuration and visualizes according to its widget configuration. These widgets are automatically updated as data is streamed at Carriots using Rest API. Thus the client application allows real time monitoring and water management. Water management using the client application involves monitoring the water levels and water quality along with water dispensation. It also includes an alert system to alert the authoritative person when the water level goes beyond a certain threshold value and when there is abnormality in the water quality. Water dispensation is implemented by finding the nearest tank with available water. When there is no sufficient amount of water in the tank, the nearest tank is determined and suggestions are given to the authoritative person notifying him/her. Thereby, the system serves as an advisor during the water crisis. The system is also capable of generating various reports based on the available sensed data. They include,

A. Amount of water in each tank, thereby calculating the total

amount of water in city.

B. Amount of water consumption per area per month.

This water level monitoring, water quality monitoring, water dispensation, generation of reports together forms the IoT based efficient water management system.

The accuracy of the system is achieved using sensors. Ultrasonic sensor provides the water level measurement with millimetre precision. It also allows the water level to be updated for every millisecond. The water quality is determined accurately as a combination of $\mathrm{PH}$, conductivity and turbidity sensors are used. Raspberry $\mathrm{Pi}$ is used as a microprocessor considering the following parameters: RAM, clock speed, input voltage required and processor. So the chosen sensors and microprocessor gives high performance to the system.

\section{F. Smart Water Monitoring System Using IOT At Home [6]}

This designed smart water system can be easily applied to home, offices, and schools and at any places where water tanks are used. By placing this system in a smart building, we will be able to collect and analyze the water usage patterns of the residents and save a lot of water from wastage. This is the small contribution from our side to save and supply good quality of water. he system can monitor water quality automatically, and it is low in cost and does not require people on duty. This system is used to avoid the huge amount of water is being wasted by uncontrolled use of home/offices etc. So the water quality testing is likely to be more economical, convenient and fast.

In water tank level monitoring system for monitoring the level of tank we use the ultrasonic sensor. An ultrasonic sensor is a device that can measure the distance by using sound waves. In water level monitoring the transmitter send the sound waves and receiver receive the signal. By using following formula we calculate the distance.

Distance $=($ speed of sound $*$ time taken $) / 2$

In our system we consider that the height of our tank is $30 \mathrm{~cm}$. To avoid the wastage of water we use the automation for the motor. If the water level is less than $20 \%$ then microcontroller send the +ve signal to the relay and relay automatically gets $\mathrm{ON}$ and motor will be get started. If the level of water tank is greater than or equal to $80 \%$ then microcontroller send the -ve signal to the relay and relay will be automatically gets OFF then motor also gets OFF. In this both the situations the GSM send the message to the user. If the level is less than $20 \%$ then it will send the message Alert: level is 20. If the level is greater than or equal to $80 \%$ then it will send the message like Alert: level is 80 .

Water quality monitoring system is very important for measure the quality of the water. To measure the quality of water we use the $\mathrm{pH}$ and the temperature sensor. The $\mathrm{pH}$ stands for "Potential of Hydrogen," referring to the amount of hydrogen found in water. $\mathrm{pH}$ is measured on a scale that runs from 0 to 14 . 7 is neutral, meaning there is a balance between acid and alkalinity. A measurement below 7 means acid is present and a measurement above 7 is basic (or alkaline). The second parameter is a temperature. Temperature will also affect the equilibrium and the $\mathrm{pH}$. In pure water, a decrease in $\mathrm{pH}$ of about 0.45 occurs as the temperature is raised by $25{ }^{\circ} \mathrm{C}$. The $\mathrm{pH}$ probes were kept and temperature sensor in the water for two minute and recorded the $\mathrm{pH}$ value and temperature value that was displayed on the meter screen on Labview. In this water quality monitoring system if the value of $\mathrm{pH}$ sensor is greater than 7 then it will be send the message to the user and if the temperature is greater than 50 then also the GSM send the message to the user.

In the water pipe leakage detection system for detecting the leakage we use the flow sensor to measure the flow of the water. In our system we use the two flow sensors for measure the flow of water. If the flow measured from first flow sensor and flow measured from second flow sensor is not equal that means the leakage is present in our system. If the leakage is occurred in system then the GSM send the message to the user that is Alert: Leakage is detected.

In this system the microcontroller is the core component which controls all the devices those are connected to the microcontroller. Ultrasonic, $\mathrm{pH}$, temperature, flow sensor, motor, 22 GSM is connected to the microcontroller. Microcontroller sends the data to the computer through the USB connector. Motor is used to fill the empty tank. If the tank level is below $10 \%$ then motor automatically gets ON and if tank level is greater than $80 \%$ then it will be gets OFF. The relay is used to control the functioning of motor. Relays are switches that open and close circuits electromechanically. Relay controls one electrical circuit by opening and closing contacts in another circuit. In model the coding of required component is written in Arduino IDE. In this the output can be shown on serial monitor. The author has connected microcontroller to the computer through USB cable and then measured the data measured by sensor. System runs on battery power and comprises of four sub circuits working synchronously; sensor circuit, controller circuit, SMS circuit and relay driver circuit. Sensor senses the level of the water in tank which is continuously fed to controller system. As the system encounters the empty level condition, status of load shedding is checked. Relay coil is energized and the pump operates when there is no load shedding. 


\section{G. IOT Based Water Monitoring System: A Review [7]}

This paper is presented the design and development of IoT based water monitoring \& control system .For this some sensors are used. The collected data from the all the sensors are used for analysis purpose for better solution of water problems. The data is sends to the cloud server via Wi-Fi module ESP8266. So this application will be the best challenger in real time monitoring \& control system and use to solve all the water related problems. This paper propose a more efficient water monitoring and control system for water utility to reduce the current water wastage problem. This approach will help utilities operators improve low cost water management systems, specially by using rising technologies and IoT is one of them. The Internet of Things (IoT) could prove to be one of the most important methods for developing more utility-proper systems and for making the consumption of water resources more efficient.

This study discusses the design and current development of system having low cost to monitor real time values and also to control the system using IoT. To measure the various parameters of the water, array of sensors are included in the system. The parameters which can be measured are like temperature, $\mathrm{PH}$, turbidity of the water. Core controller can process the value measured from the sensors. The Arduino Uno model can be used to control the system. Lastly, to access the sensor data on internet, cloud computing can be used.

Relation with IOT: In past, the living of individuals has been changed due to the Internet. The IoT has been became an emerging research area because of need of an establishment for connecting things, sensors and other smart technologies. IoT is known as internet's advanced version. Information related to physical objects can be immediately accessed by IoT and results into novel system having high efficiency and outputs. In IoT, a number of main technologies are there like ubiquitous computing, RFIP, wireless sensor network, cloud computing. Cloud computing, a large-scale, low cost processing unit and also an IP based connection mostly used for calculation and storage purpose. The water quality monitoring application contains many distributed monitoring sensors' array and a wide distribution network. Separate monitoring system is also required in it as told in paper. This paper introduces cloud computing techniques for screening values on the internet.

The hardware of the proposed system consists of Arduino microcontroller development board, Ultrasonic Sensors, Turbidity sensor, $\mathrm{pH}$ sensor ,Wi-Fi module to collect \& transfer data to cloud. Data is collected from different types of sensors mentioned above by using Arduino Uno microcontroller. Arduino is a open source hardware platform which is able to work with various sensors and communication technology. There are different types of Arduino microcontroller that are used for different purpose. It not only control devices but also can read data from all types of sensor. It is simple, low cost and easy to use. It takes $5 \mathrm{~V}$ voltage as input speed $16 \mathrm{MHz}$.Arduino Uno contains the 14 digital i/o pins and 6 analog input pins to connect various sensors that gives analog inputs. The Raspberry $\mathrm{Pi}$ is a Quad-Core 64bit CPU, WiFi and Bluetooth. Raspberry Pi 3 Is the 3 Raspberry Pi[13]. It is a credit card sized board computer which is used for multiple applications. Although maintaining the popular board format the Raspberry Pi 3 Model B brings you a more powerful processor, 10x faster than the first generation Raspberry Pi. Additionally it adds wireless LAN \& Bluetooth connectivity making it the ideal solution for powerful connected.

This system will be built using Arduino Uno and Node MCU. Arduino Uno is connected with Water level sensor(HCSRO4), Turbidity Sensor, pH sensor, Wi-Fi module (ESP8266) that process and transfer sensed data to cloud. . And other side ultrasonic sensor connected with Node MCU. This stored data is accessed by users. This enables the user to check the level of water and if it goes full then automatic stop. Other parameters related to water like water quality can also monitored for prevent wastage of water.

\section{H. Water Management System Using IOT [8]}

This paper presents an IoT device which helps to manage and plan the usage of water. This system can be easily installed and maintained for long run. The Laser sensor is placed on the tank which continuously monitors the water level in real time. This information will be updated in the cloud and user can analyze the amount of water. According to the level of water in the tank, the motor functioning is automatically controlled. When the water level falls below the threshold level the motor will be again turned on automatically. Overflowing water tanks in residence, schools, colleges, Municipal overhead tanks, Hospitals etc. can contribute to the massive amount of water wastage. If we can control this we can save large amounts of water. Conventional water tanks can neither monitor nor control the water level in the tank. As of now, the water level has to be manually checked and refilled according to the requirements.

So in this paper, the above mention problems are solved with automatic water level detection and refilling of water storage system with the help of Internet of Things (IoT). Presented here is a Water Management System using IoT. Water level indication, automatic water pump on/off, etc are carried out by this project.

Laser sensor used in this project is VL53LOX for precise level indication. The issue of water scarcity is becoming more prevalent. The IoT enabled water management solutions like this use sensor to collect data and share data to the cloud.

The transmitter section consists of an Arduino, HC12 transmitter, laser sensor, and NodeMcu. In the automatic water level detection and refilling of water storage system, the sensor used is Laser sensor which is a replacement of ultrasonic sensor because of its accuracy and small size.

The Laser sensor is used to detect the water level. The Laser sensor is placed above the tank which continuously monitors the water level in real time. This information will be updated in the cloud and user can analyze the amount of water. These sensor values are sending to water pump via the HC12 transmitter to turn on/off the pump. The sensor values are also forwarded to NodeMCU which is used for the IoT purpose. NodeMCU connects the system to a cloud storage. Here we use Adafruit cloud platform. The platform is designed in such a way that it will show the instantaneous value of current status of water. The water level measured 
by sensors is sent continuously to NodeMcu and forwarded to Adafruit cloud, it gives a graphical representation of water level from which we can analyze our water usage.

The receiver section consists of Arduino Uno, relay, $\mathrm{HC} 12$ receiver and a motor. According to the value received from the sensors about water level to HC 12 receiver, the motor will automatically turn on/off to pump the water to the tank. Depending on the water levels, as described above, the status of the motor will be automatically controlled. If the water level is in between maximum and minimum level set, then the user can control the status of the motor from the Adafruit cloud platform. Buttons ON and OFF have been provided for the same.

In the automatic water level detection and refilling of water storage system, the sensor used is Laser sensor which is a replacement of ultrasonic sensor because of its accuracy and small in size. The sensor is placed on top of the tank facing downwards. The Laser sensor is used to detect the water level. Nowadays liquid level monitoring is vital in many industries too like oil, automotive etc. Using our smart system we can analyse the usage and also detect the leakage in the tanks of these industries.

\section{IOT Based water Management System For Smart City [9]}

This paper will demonstrate the successful implementation of an internet-based approach to measuring water quality and usage on a real-time basis. A flow sensor for measuring of quantity supplied, eliminating the drawbacks of traditional water metering systems. Future enhancements can include prepaid billing and automatic treatment of water based on the nature of contamination. Water metering system will be used for automated billing, eliminating the drawbacks of traditional water metering systems. This novel idea can be further extended to other areas like oil and natural gas monitoring systems. During the past decade, water needs have increased unpredictably in India. Increasing demand of water supply has become a major challenge for the world. Wasteful usage of water, climatic changes and Urbanization has further depleted the resource. Conservation and management of the resource must be given utmost importance. In this paper, we present an IoT design for water monitoring and control approach which supports internet based data collection on real time bases. The system addresses new challenges in the water sector -flow rate measuring and the need for a study of the supply of water in order to curb water wastage and encourage its conservation. We also measure the quality of water distributed to every household by

deploying $\mathrm{pH}$ and conductivity sensors. The traditional water metering systems require periodic human intervention for maintenance making it inconvenient and often least effective. For shortcoming of the existing models for a ubiquitous usage of

wireless systems for smart quality monitoring and communicate data wirelessly.

This system can be implemented on water tanks for safe and waste less consumption. Water when supplied from the reservoir to tanks then the $\mathrm{pH}$ level of water will be checked, if it comes in required range than the conductivity of water will be checked. If $\mathrm{pH}$ or conductivity of water will not be in safe range than the water will not be supplied to household tanks and valves will be closed. The Same procedure will be followed till water does not come in safe range. After the satisfactory quality check of water if the tanks are full than valves of the tank will be opened and water will be distributed. During distribution of water rate of flow is measured so that equal distribution is done. This whole data is sent from Wi-Fi to the Web page so that system can be accessed remotely from a computer. The flow of distribution and quality of water both will be monitored from the web page which can be displayed anywhere using the internet.

Electrical conductivity is also an indicator of water quality. It measures free chlorine without sample pretreatment. It does not have messy and expensive reagents needed. Conductivity data can detect contaminants, determine the concentration of solutions and determine the purity of water. It is Compact in size. Conductivity sensor measures conductivity by $\mathrm{AC}$ voltage applied to nickel electrodes. These electrodes are placed in a water sample and reading is obtained.

\section{EXISTING SYSTEM}

In the existing system, it starts with the distribution of water in the city with the help of stepper motor receiving signals from microcontroller for ON/OFF, leakage detection is carried out by pressure sensors and quality parameters like $\mathrm{pH}$, temperature, turbidity are measured through using different sensors like $\mathrm{pH}$ sensors, temperature sensors, conductivity sensors, etc. And at the tank level of water is measured by using ultrasonic sensor and indicating the level by using the led lights.

The drawbacks of the system:

The notification is not sent to the government officials \& users about the distribution of water, leakage is not effectively detected in pipes. The system is partially implemented \& not that effective.

\section{CONCLUSION}

This paper presents the design and development of real time water monitoring system with all the advanced methodologies. Additionally, a combination of all these advancements is not an

impossible task and can be effectively completed. Looking towards further modifications in case of more enhancements, the data stored from Smart System analysis can be used as a platform for

future plans and new strategies at any instance of time via smart terminals connected. Further changes will be made to make this system more cost effective and more firm for deploying in different areas.

\section{ACKNOWLEDGEMENT}

This work is partially supported by Department of Electrical \& Electronics Engineering, BMS Institute Of Technology \& Management, Yelhanka, Bangalore. We offer our sincere gratitude to Prof. Dr. N Ramarao (Project Coordinator \& Head of Department Electrical \& Electronics) BMSIT \& M, Bangalore who guided us and supported us. We take this opportunity to express gratitude to all department faculty members for their help support. 


\section{REFERENCES}

[1]. Mrs. Sarswathi V, Internatioal Journal of Innovative Research in Engineering and Management (IJIREM), volume-5, issue-2, march-2018.

[2]. Anirudh Das.B, K.S.Srivatsava, Pradeep Doss. "Water utiliry monitoring and control using IOT based approach" IJECT volume9, Issue-2, april-june 2018

[3]. Joy shah, International journal of innovative research in science,engineering and technology. Volume-6, Issue-3, march2017.

[4]. Sangeeta sarkar, Susmitha sikder, Saiful islam ashik, Ayesha siddika, Global scientific journals, GSJ: volume-6, Issue-10, october 2018

[5]. N.Yazhini, Faustina Joan S P, International conference on advancements in computing technologies, ICACT 2018: volume-4, Issue-2

[6]. M.B.Kawarkhe, Sanjay Agarwal, IOSR journal of computer engineering (JCE): volume-21, Issue-1, Ser.II(jan-feb 2019)

[7]. Pragati.Damor, Kirtikumar J Sharma, International Journal of advance engineering and reseach development, volume-4, Issue-6, June 2017.

[8]. Jeny Joseph, Manju K M, Sajith M R, Sujith nair, Vishnu P Viay, Sitara Krishnan, International research journal of engineering and technology(IRJET). Volume-05, Issue-04, April 2018

[9]. H.Amatulla Patawala, International journal of Advance research, ideas and innovation in technology: volume-3, Issue-2 